\title{
A patient with shoulder and lumbar pain (2009: 6a)
}

(C) European Society of Radiology 2008

\section{Case report}

A 76 year-old, non-smoking woman presented with a 3-month history of intermittent right lumbar pain radiating to the right shoulder, increasing on deep inspiration. The patient's past medical history was characterized by mild hypertension and gastro-esophageal reflux. Physical examination and routine laboratory investigations were unremarkable.

A multi-detector CT study was performed and thin section axial images before (Fig. 1) and after intravenous injection of contrast medium (Fig. 2 arterial phase) are presented.

\section{What is the diagnosis?}

Readers are invited to supply one possible diagnosis via electronic means to: robert.hermans@uzleuven.be
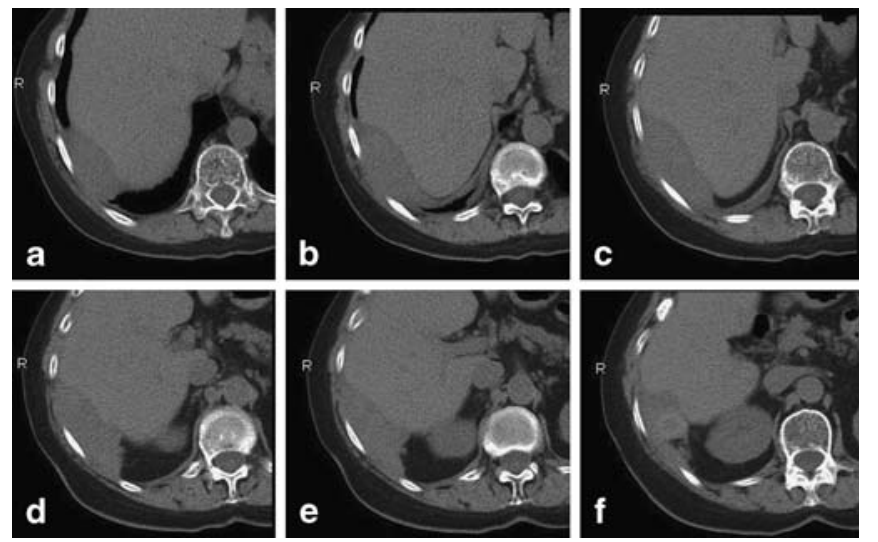

Fig. 1a-f Unenhanced CT images, from a (cranial) to $\mathbf{f}$ (caudal)
The subject of the email should include 'Interpretation Corner' and the number given above (2008: 6a). You should include your name, title, address, fax and phone number.

Deadline: one clear calendar month from distribution date.

Three months after the initial publication of the case history, the authors will publish the final diagnosis and a brief summary. The summary will describe exactly how the case was investigated at the host institution, how the diagnosis was established and the teaching points of the case in question.

The names of the first 25 radiologists submitting the correct diagnosis will be published (only one from any individual centre and none from the host institution!)
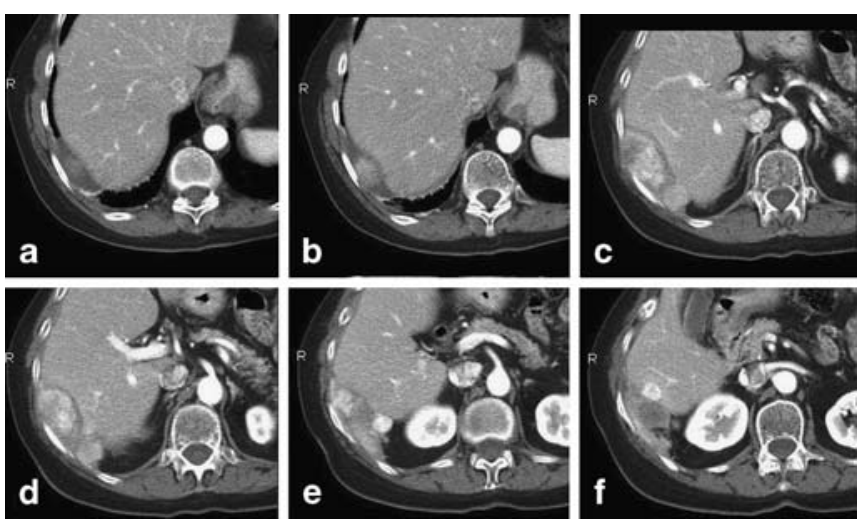

Fig. 2a-f CT images after injection of contrast agent (arterial phase), from a (cranial) to $\mathbf{f}$ (caudal) 